\title{
HARMONIC FUNCTIONS OPERATING IN HERMITIAN BANACH ALGEBRAS
}

\author{
A. El Kinani
}

\begin{abstract}
The purpose of this paper is to introduce a harmonic functional calculus in order to generalize some extended versions of theorems of von Neumann, Heinz and Ky Fan.
\end{abstract}

\section{Introduction and notations}

Let $A$ be an algebra over the complex field with the involution $*$ and unit $e$. The symbols $\operatorname{Sp} x$ and $\rho(x)$ denote the spectrum of $x$ and the spectral radius of $x$, respectively. An element $x$ in $A$ is said to be hermitian if $x=x^{*}$. An element $x$ in $A$ is said to be unitary if $x x^{*}=x^{*} x=e$. Denote by $U$ the set of all unitary elements of $A$, by $U_{e}$ the identity component in $U$. We say that the algebra $A$ is hermitian if the spectrum of every hermitian element of $A$ is real. As in [5], we denote by $|x|_{*}$ the square-root of the spectral radius of the element $x^{*} x$, i.e., $|x|_{*}=\rho\left(x^{*} x\right)^{\frac{1}{2}}$.

Let $\Omega$ be an open subset of C. An $A$-valued function $f: \Omega \rightarrow A$ is said to be holomorphic on $\Omega$ if $\varphi(f(z))$ is holomorphic on $\Omega$ in the classical sense for every $\varphi \in A^{\prime}$, where $A^{\prime}$ denotes the conjugate space of $A$. We will denote by $H(\Omega, A)$ the set of all holomorphic $A$-valued functions on $\Omega$. Let $f: \Omega \rightarrow A$ be a $C^{(2)}$ function of two real variables $x$ and $y$. Recall that $f$ is said to be harmonic if it satisfies the differential equation: $\frac{\partial^{2} f}{\partial x^{2}}+\frac{\partial^{2} f}{\partial y^{2}}=0$ on $\Omega$. The set of all harmonic $A$-valued functions on $\Omega$ is denoted by $h(\Omega, A)$. For the scalar functions, we denote simply: $H(\Omega)=H(\Omega, C)$ and $h(\Omega)=h(\Omega, C)$.

Keywords. Hermitian algebra, harmonic functional calculus, Unitary element, Topological proper contraction.

1991 Mathematics subject classifications: Primary: 46 K 99; Secondary: 46 H 30. 
Throughout the paper $e$ will denote the unit of $A$ (not necessarily of norm 1), and for the complex number $z$ we often simply write $z$ instead of $z e$. Also the open unit disk and the closed disk will be denoted by $D$ and $\bar{D}$ respectively.

In [7], K. Fan proves the following:

Theorem (K. Fan). Let $f$ be a complex function analytic on the open unit disk $D$. If $f(D) \subset D$, then the inequality $\|f(T)\|<1$ holds for every operator $T$ on a Hilbert space $H$ with $\|T\|<1$.

It was pointed out in [7] that K. Fan's Theorem is closely related to the important theorem of von Neumann given also in [7]. We have extended, in [3], Fan's Theorem to hermitian Banach algebras. In [10], Shih and Tan introduced a Hilbert space norm equivalent to the original one and proved the analog of Fan's Theorem for commuting topological proper contractions. Generalizations in two settings of the results of [10] to analytic vector valued functions in hermitian algebras are obtained in [5] and [6].

In this paper, we extend K. Fan's Theorem to harmonic functions in hermitian algebras. An analog of von Neumann's inequality and two theorems on the maximum principle are obtained. We also make an extension of Shih and Tan's Theorem.

\section{Some preparatory results}

The functional calculus for harmonic $A$-valued functions was defined in $[\mathbf{4}]$ and $[\mathbf{5}]$. Let us recall the following definition.

Definition 2.1. Let $A$ be a complex unital Banach algebra with continuous involution, $\Omega$ an open subset of $C, z_{0} \in \Omega$ such that $\overline{D\left(z_{0}, R\right)} \subset \Omega$ $(R>0), x$ an element of $A$ with $\operatorname{Sp} x \subset D\left(z_{0}, R\right)$ and $f \in h(\Omega, A)$. Then

$$
f(x)=\frac{1}{2 \pi} \int_{\left|z-z_{0}\right|=R} f(z) \operatorname{Re}\left[\left(z+x-2 z_{0}\right)(z-x)^{-1}\right] \frac{|d z|}{R} .
$$

The following lemmas will be needed later on.

Lemma 2.2. Let $A$ be a complex unital Banach algebra with continuous involution. Assume that $f \in h\left(\Omega_{1}\right)$ and $g \in H\left(\Omega_{2}, A\right)$ satisfy the condition that for each compact set $K$ in $\Omega_{2}$, there exists a disk $\overline{D\left(w_{0}, R\right)} \subset \Omega_{1},(R>0)$, such that $\operatorname{Sp} g(z) \subset D\left(w_{0}, R\right)$ for every $z \in K$. 
Then the function $h$ defined by $h(z)=f(g(z))$ for $z \in \Omega_{2}$ is an element of $h\left(\Omega_{2}, A\right)$.

Proof: Let $z_{0} \in \Omega_{2}$ and $r>0$ such that $\overline{D\left(z_{0}, r\right)} \subset \Omega_{2}$. Consider the set $\Gamma_{2}=\left\{z \in C:\left|z-z_{0}\right|=r\right\}$ and $\overline{D\left(w_{0}, R\right)}$ be a disk in $\Omega_{1}$ such that $\operatorname{Sp} g(z) \subset D\left(w_{0}, R\right)$ for $\left|z-z_{0}\right| \leq r$. Put $\Gamma_{1}=\left\{w \in C:\left|w-w_{0}\right|=R\right\}$. Then for $z \in D\left(z_{0}, r\right)$, we have

$$
h(z)=f(g(z))=\frac{1}{2 \pi} \int_{\Gamma_{1}} f(w) \operatorname{Re}\left[\left(w+g(z)-2 w_{0}\right)(w-g(z))^{-1}\right] \frac{|d w|}{R} .
$$

Since for any fixed $w$ on $\Gamma_{1}$ the function $k$ defined by

$$
k: z \rightarrow \operatorname{Re}\left[\left(w+g(z)-2 w_{0}\right)(w-g(z))^{-1}\right]
$$

is harmonic on $D\left(z_{0}, r\right)$ and continuous on $\Gamma_{2}$, it follows that:

$$
k(z)=\frac{1}{2 \pi} \int_{\Gamma_{2}} k(u) \operatorname{Re}\left[\left(u+z-z_{0}\right)(u-z)^{-1}\right] \frac{|d u|}{r},
$$

which shows that

$$
\left.h(z)=\left(\frac{1}{2 \pi}\right)^{2} \int_{\Gamma_{1}} \int_{\Gamma_{2}} f(w) k(u) \operatorname{Re}\left[\left(u+z-2 z_{0}\right)(u-z)\right)^{-1}\right] \frac{|d u|}{r} \frac{|d w|}{R}
$$

for every $z \in D\left(z_{0}, r\right)$. Let $\varphi$ be any bounded linear functional on $A$. A simple verification will show $\varphi(h(z))$ is harmonic on $D\left(z_{0}, r\right)$ and so is $h$. This completes the proof.

If $S$ is a subset of $A$, let co $S$ denote the convex hull of $S$, and $\overline{\operatorname{co} S}$ its closure. Put $A_{1}=\left\{x \in A:|x|_{*}<1, \rho(x)<1\right\}$. For any $x \in A_{1}$, the Möbius transformation $\Phi_{x}$ is defined by:

$$
\Phi_{x}(\lambda)=\left(e-x x^{*}\right)^{-\frac{1}{2}}(\lambda+x)\left(e+\lambda x^{*}\right)^{-1}\left(e-x^{*} x\right)^{\frac{1}{2}}, \quad \lambda \in C
$$

satisfying $|\lambda|<\rho(x)^{-1}$. It is clear that $\Phi_{x}$ is holomorphic in a neighborhood of $\bar{D}$. Moreover, $\Phi_{x}(0)=x$, and maps the unit circle into $U_{e}$ [8, p. 2].

We now prove the following version of the maximum principle.

Lemma 2.3. Let $A$ be a complex unital Banach algebra with continuous involution, and $\Omega$ be an open subset of $C$ containing $\bar{D}$. If $f \in h(\Omega)$, then

i) $\|f(x)\| \leq \operatorname{Sup}\left\{\|f(y)\|: y \in U_{e}\right\} \leq \infty$ for all $x$ in $A_{1}$ satisfying $\operatorname{Sp} \Phi_{x}(\lambda) \subset \bar{D}$ for all $|\lambda|=1$,

ii) If $A$ is hermitian, then $|f(x)|_{*} \leq \operatorname{Sup}\left\{|f(y)|_{*}: y \in U_{e}\right\} \leq \infty$ for every $x$ in $A_{1}$. 
Proof: i) Observe first that $\rho\left(\Phi_{x}(\lambda)\right)$ is a subharmonic function of $\lambda$ in a neighborhood of $\bar{D}$. Then, by the maximum modulus principle, we have $\operatorname{Sp} \Phi_{x}(\lambda) \subset \bar{D}$ for all $|\lambda| \leq 1$. Since $\Omega$ is a neighborhood of $\bar{D}$, it follows that $f\left(\Phi_{x}(\lambda)\right)$ is defined for all $|\lambda| \leq 1$. Define for any $\varphi \in A^{\prime}, F(\lambda)=$ $\operatorname{Re}\left(\varphi\left(f\left(\Phi_{x}(\lambda)\right)\right)\right)$. Suppose $K$ is a compact subset of $D$. Again by the maximum modulus principle we see that $\max \left\{\rho\left(\Phi_{x}(\lambda)\right): \lambda \in K\right\}=r<1$ and hence $\operatorname{Sp} \Phi_{x}(\lambda)$ is contained in $\overline{D(0, r)}$ for all $\lambda \in K$. Thus, by Lemma 2.2, $F$ belongs to $h(D)$. Furthermore $F$ is continous in $\bar{D}$. It follows from the classical maximum principle that $F(0) \leq \operatorname{Sup} F(\lambda)$ and consequently $\operatorname{Re} \varphi(f(x)) \leq \operatorname{Sup} \operatorname{Re} \varphi\left(f\left(U_{e}\right)\right)$. Therefore, by a separation theorem [2, p. 417], $f(x) \in \overline{\operatorname{Co}} f\left(U_{e}\right)$; whence i). Concerning ii), $|\cdot|_{*}$ is a continuous algebra pseudonorm on $A$ such that $\rho(x) \leq|x|_{*}$ for all $x \in A$. For all $|\lambda|=1$, we have $\left|\Phi_{x}(\lambda)\right|_{*}=1$. As above $f(x) \in \overline{\operatorname{Co}} f\left(U_{e}\right)$ and ii) holds.

\section{Some inequalities for harmonic functions}

In the sequel $A$ will denoted a complex unital hermitian Banach algebra with continuous involution. The following result generalizes von Neumann's Theorem [3, p. 934].

Theorem 3.1. Let $f$ be a harmonic function on some neighborhood of $\bar{D}$ and $x \in A$ with $|x|_{*} \leq 1$. If $|f(z)| \leq 1$ for $z \in \bar{D}$, then $|f(x)|_{*} \leq 1$.

Proof: By Lemma 2.3, it suffices to show that the number $r=$ $\operatorname{Sup}\left\{|f(y)|_{*}: y \in U_{e}\right\}$ satisfies $r \leq 1$. Let $y \in U_{e}$. Since $\rho(y)=|y|_{*}=1$, it follows that $\operatorname{Sp} y \subset \bar{D}$. Thus $f(y)$ is defined. Moreover using Definition 2.1, one can prove that $f(y)$ is a normal and hence $|f(y)|_{*}=\rho(f(y))$. Finally, by the spectral mapping theorem $([\mathbf{1}]), \operatorname{Sp} f(y)=f(\operatorname{Sp} y)$. Thus $|f(y)|_{*} \leq 1$.

A consequence of Theorem 3.1 is the following:

Theorem 3.2. Suppose $f \in h(D)$. For $0<r<1$, let $M(r)=$ $\underset{|z|=r}{\operatorname{Max}}|f(z)|$. Then $M(r)=\operatorname{Max}_{|x|_{*} \leq r}|f(x)|_{*}$.

Proof: If $M(r)=0$, then the theorem is trivially true. Assume that $M(r)>0$. The function $g$ defined by $g(z)=f(r z) / M(r)$ is harmonic on $|z|<r^{-1}$. By the maximum modulus principle, $|g(z)| \leq 1$ for $z \in \bar{D}$. An application of Theorem 3.1 to $g$ shows that $|g(x)|_{*} \leq 1$ for every $x \in A$ with $|x|_{*} \leq 1$. This is equivalent to saying that $|f(x)| \leq M(r)$ for $|x|_{*} \leq r$ 
and $\operatorname{Max}_{|x|_{*} \leq r}|f(x)|_{*} \leq M(r)$. Furthermore, if $\left|z_{0}\right|=r$ and $\left|f\left(z_{0}\right)\right|=M(r)$, then $\left|f\left(z_{0} e\right)\right|_{*}=\left|f\left(z_{0}\right) e\right|_{*}=\left|f\left(z_{0}\right) e\right|_{*}=\left|f\left(z_{0}\right)\right|=M(r)$. This completes the proof.

A direct consequence of Theorem 3.2 is the following result which clearly generalizes Theorem 3.1 of $[\mathbf{3}]$.

Theorem 3.3. Let $x \in A$ with $|x|_{*}<1$, and $f \in h(D)$ such that $f(D) \subset D$. Then $|f(x)|_{*}<1$.

Remark 3.4. Theorem 3.3 is derived from Theorem 3.1. Using the proof of Theorem 2 of [4] and the continuity of the harmonic calculus $([\mathbf{1}])$, one can prove that the two theorems are equivalent.

Recall [5, p. 199] that an element $x$ of $A$ is said to be a topological proper contraction if $\rho(x)<1$. If $\rho(x) \leq 1, x$ is said to be a topological contraction. Using Theorem 3.3 and Lemma III.10 of [5], one can prove the following:

Theorem 3.5. Let $x \in A$ be a topological proper contraction. Then there exists a hermitian involution \# on A such that:

1) $\mid$ * is equivalent to $\mid$ |\#.

2) $|f(x)|_{\#}<1$ for every $f \in h(D)$ such that $f(D) \subset D$.

Another statement that is intimately related to Theorem 3.5 is the following:

Theorem 3.6. Consider $\delta>0$ and $\Omega=\{z:|z|<1+2 \delta\}$. If $x \in A$ is a topological contraction, then there exists a hermitian involution \# on $A$ such that

1) ||$_{*}$ is equivalent to $\mid$ |\#.

2) $|f(x)|_{\#} \leq 1$ for every $f \in h(\Omega)$ with $|f(z)| \leq 1$ for all $z \in \Omega$.

Appealing to Theorem 3.5, we obtain another version of the maximum principle, the first version of which was proved in Theorem 3.2. 
Theorem 3.7. If $f \in h(D)$, then for $0<r<1, \max \{|f(z)|:|z| \leq$ $r\}=\underset{\rho(x) \leq r}{\operatorname{Max}} \rho(f(x))$.

Proof: As in the proof of Theorem 3.2, we suppose $M(r)>0$. The function $g$ defined by $g(z)=f(r z) / M(r)$ is harmonic on $|z|<r^{-1}$. By the maximum modulus principle, $|g(z)|<1$ for all $z \in D$. If $x \in A$ with $\rho(x)<1$, by Theorem 3.5 there exists a hermitian involution \# on $A$ such that $|g(x)|_{\#}<1$ and then $\rho(g(x))<1$. Therefore $\rho(f(r x)) \leq M(r)$ for $\rho(x)<1$ which is equivalent to $\rho(f(x)) \leq M(r)$ for $\rho(x) \leq r$. Thus $\max _{\rho(x) \leq r} \rho(f(x)) \leq M(r)$. Moreover if $\left|z_{0}\right|=r$ and $\left|f\left(z_{0}\right)\right|=M(r)$ then $\rho\left(z_{0} e\right)=\left|z_{0}\right|=r$ and $\rho\left(f\left(z_{0} e\right)\right)=\rho\left(f\left(z_{0}\right)\right)=M(r)$ and we are done.

Acknowledgements. The author would like to express his thanks to Professor M. Oudadess for his comments and his advice.

\section{References}

1. M. Akkar, A. El Kinani and M. Oudadess, Calculs fonctionnels harmonique et analytique réel, Ann. Sci. Math. Québec 12 (1988), 151-169.

2. N. Dunford AND J. T. SChwartz, "Linear operators," Interscience, New York, p. I, 1958; p. II, 1963.

3. A. El KinAni, Holomorphic functions operating in hermitian Banach algebras, Proc. Amer. Math. Soc. 111 (1991), 931-939.

4. A. EL KinAnI, Fonctions harmoniques opérant sur les algèbres de Banach involutives, Ann. Inst. Fourier (Grenoble) 41 (1991), 493-509.

5. A. El Kinani, Fonctions analytiques de contractions topologiques dans les algèbres hermitiennes, Collect. Math. 41 (1990), 197-216.

6. A. El Kinani, Calcul harmonique dans les algèbres de Banach hermitiennes, Rend. Circ. Mat. Palermo XLII (1993), 257-272.

7. K. FAn, Analytic functions of a proper contraction, Math. Z. 160 (1978), 275-290.

8. E. Heinz, Ein von Neumannscher satz über beschränkte operatoren in Hilbertschen Raum, Nachr. Akad. Wiss. Göttingen Math.-Phys. Kl. II (1953), 5-6.

9. L. HARRIS, Banach algebras with involution and Möbius transformation, J. Funct. Anal. 11 (1972), 1-16. 
10. M. H. SHIH AND K. K. TAN, Analytic fonctions of topological proper contractions, Math. Z. 187 (1984), 317-323.

Ecole Normale Supérieure Takaddoum

B.P. 5118

10105 Rabat

MAROC

Primera versió rebuda el 4 de Març de 1996, darrera versió rebuda el 26 de Febrer de 1997 